\title{
Hypo-angiogenesis: A possible pathological factor in the development of dry age-related macular degeneration and a novel therapeutic target
}

\author{
Pradeep Venkatesh ${ }^{1}$ \\ ${ }^{I}$ Rajendra Prasad Centre for Ophthalmic Sciences, All India Institute of Medical Sciences Sciences (AIIMS), New Delhi, India
}

\begin{abstract}
Background: Angiogenesis causes severe vision loss in patients with exudative or wet forms of age-related macular degeneration (AMD). The pathogenesis involves upregulation of several proangiogenic factors, particularly the vascular endothelial growth factor (VEGF). Contrary to the pathogenesis of exudative AMD, molecular events leading to the development of dry AMD remain unclear. Dry AMD is characterized by loss of the retinal pigment epithelium (RPE). The mechanism that triggers RPE cell loss remains unclear. Choriocapillaris development is absent in mice with RPE-specific deletion of VEGF. Moreover, in later life, background VEGF secretion promotes the survival of the RPE and maintains choriocapillaris integrity.

Hypothesis: We hypothesized that reduced synthesis of VEGF (hypo-angiogenesis) or abnormalities in its receptors, VEGF receptor-1 (VEGFR1) and VEGFR2, may be involved in the pathogenesis of non-exudative AMD or dry AMD. If the concept of hypo-angiogenesis as a driver for dry AMD is proven, treatment with VEGF or induction of angiogenesis could be considered. Similar attempts at therapeutic angiogenesis have been actively investigated in cardiac and limb ischemia.

Conclusions: The reasons for a patient developing exudative AMD or dry AMD remain poorly understood. Nevertheless, targeting increased VEGF production in patients with exudative AMD using anti-VEGF drugs is highly efficacious in preserving vision. Similarly, dry AMD may be a manifestation of reduced VEGF synthesis (hypo-angiogenesis) and subsequent decreased RPE cell survival. Experimental studies exploring the possibility of reduced VEGF secretion and/or increased receptor resistance/abnormality could pave the way for clinical trials of angiogenesis to treat dry AMD.

\section{KEYWORDS}

hypo-angiogenesis, angiogenesis, VEGF, vascular endothelial growth factor, age-related macular degeneration, dry AMD, exudative AMD, anti-VEGF, aflibercept, ranibizumab, bevacizumab, brolucizumab, wet macular degeneration, geographic atrophy, macular degeneration, therapeutic angiogenesis
\end{abstract}

\section{INTRODUCTION}

Pathological angiogenesis is defined as the aberrant initiation, propagation, and proliferation of abnormal vascular elements in response to prolonged oxidative stress and long-standing tissue hypoxia [1]. Angiogenesis causes severe vision loss in patients with proliferative vascular retinopathies, such as diabetic retinopathy, retinal

Correspondence: Pradeep Venkatesh, Professor, Surgical and Medical Retina, 482, Fourth floor, RP Centre for Ophthalmic Sciences, All India Institute of Medical Sciences, New Delhi- 110029, India. Tel: 01126593139, 01126593101. Email: venkyprao@yahoo.com ORCID iD: https://orcid.org/0000-0002-3706-7407

How to cite this article: Venkatesh P. Hypo-angiogenesis: A possible pathological factor in the development of dry age-related macular degeneration and a novel therapeutic target. Med Hypothesis Discov Innov Ophthalmol. 2021 Winter; 10(4): 185-190. https://doi.org/10.51329/mehdiophthal1437

Received: 24 November 2021; Accepted: 20 December 2021

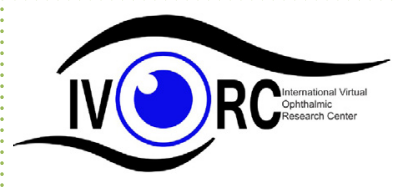

Copyright (C) Author(s). This is an open-access article distributed under the terms of the Creative Commons Attribution-NonCommercial 4.0 International License (http://creativecommons.org/licenses/by-nc/4.0/) which permits copy and redistribute the material just in noncommercial usages, provided the original work is properly cited. (c) (i) (s) 
vascular occlusion, retinal vasculitis, and hereditary vitreoretinopathies [1], and exudative or wet forms of age-related macular degeneration (AMD) [2]. The pathogenesis of AMD involves the upregulation of several proangiogenic factors, particularly the vascular endothelial growth factor (VEGF) [1]. Anti-VEGF drugs, such as aflibercept, ranibizumab, bevacizumab, and brolucizumab, are safe and effective treatments of choice for the neovascular membrane, which is characteristic of exudative AMD [2-4].

Contrary to the pathogenesis of exudative AMD, molecular events leading to the development of dry AMD remain unclear [4]. Dry AMD is characterized by loss of the retinal pigment epithelium (RPE) and advances to geographic atrophy (GA). The mechanism that triggers RPE cell loss remains unclear [4]. Factors associated with dry AMD include complement activation, mitochondrial dysfunction, inflammation, oxidative stress, and upregulation of apoptosis [5]. Recent therapeutic efforts to target the complement pathway to arrest the progression of GA have not been successful [6].

The biological effects of VEGF include vasculogenesis, angiogenesis, and increased vascular permeability. Moreover, VEGF promotes survival of cells in both vascular and non-vascular tissues [7]. It plays a critical role in the induction, differentiation, maturation, and maintenance of the RPE-choriocapillaris complex [8]. The RPE and choriocapillaris start interacting in the early embryonic period, and VEGF is a mediator of this molecular cross-talk. VEGF is responsible for the differentiation of mesenchymal cell precursors into endothelial cells of the choriocapillaris and directional distribution of the choriocapillaris fenestrations (toward the RPE) [8, 9]. The RPE is an important source of VEGF secretion. The effects are mediated by both autocrine and paracrine mechanisms; however, paracrine VEGF may not compensate for autocrine VEGF [10].

\section{HYPOTHESIS}

We hypothesized that reduced synthesis of VEGF (hypo-angiogenesis) or abnormalities in its receptors, VEGF receptor-1 (VEGFR1) and VEGFR2, may be involved in the pathogenesis of non-exudative AMD or dry AMD. The cause of reduced VEGF secretion may be secondary to oxidative stress-related damage to the nuclear and cytoplasmic components (ribosomes, endoplasmic reticulum, etc.) required for the transcription and secretion of VEGF. Reduced VEGF synthesis and/or increased VEGFR resistance impedes cell survival and induces RPE dysfunction. In early stages, this leads to the accumulation of unscavenged photoreceptor debris with subsequent activation of complement and subclinical inflammatory pathways (manifesting as drusen) [11, 12]. This, in turn, may induce the upregulation of pro-apoptotic mechanisms. With RPE dysfunction and loss, the choriocapillaris is lost because of cessation of the VEGF-mediated paracrine benefits of the RPE on the choriocapillaris.

Hypo-angiogenesis causing dry AMD is also evidenced by macular atrophy occurring as a side effect of sustained anti-VEGF treatment for exudative AMD (Figure 1) [13-16]. The role of hypo-angiogenesis in GA should be investigated to pave the way for angiogenesis as a treatment modality.
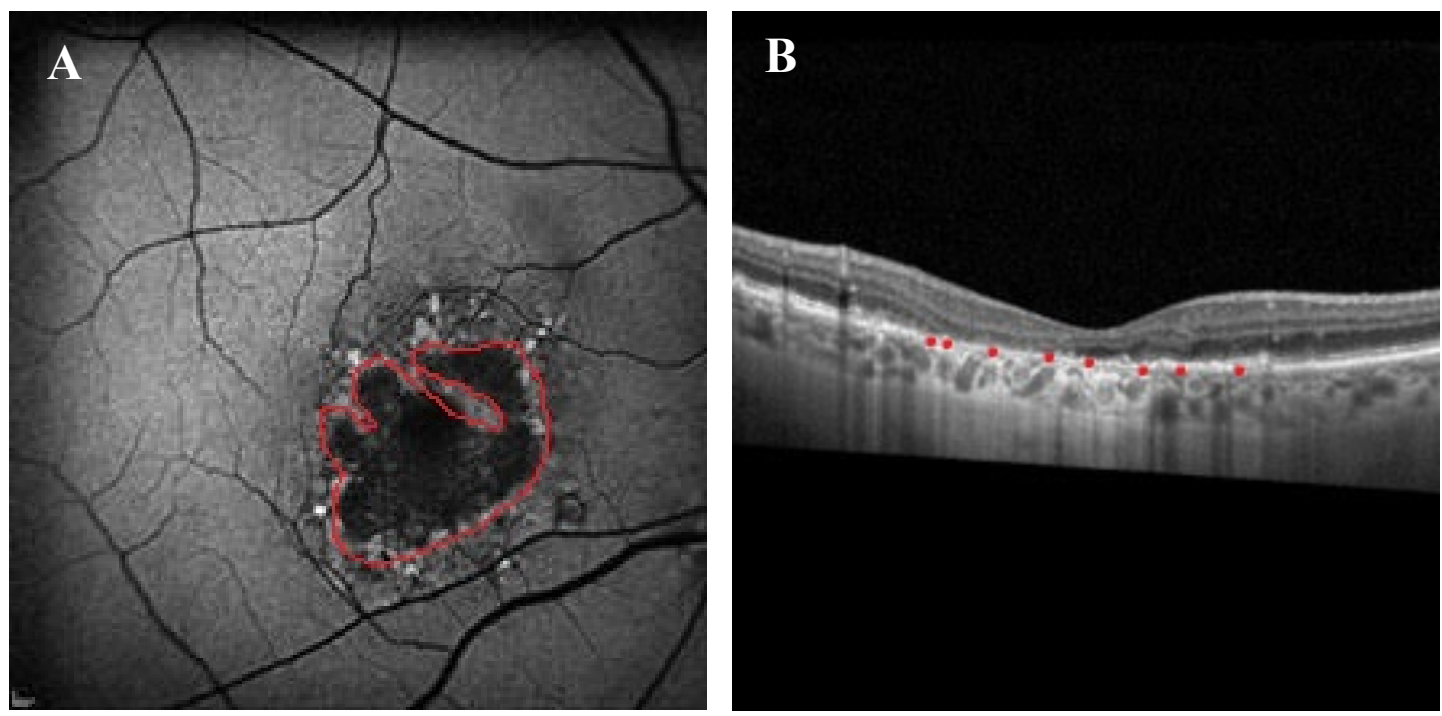

Figure 1. (A) Fundus autofluorescence in a patient with exudative age-related macular degeneration in the right eye, who received five intravitreal injections of the anti-vascular endothelial growth factor, showing a large hypo-autofluorescent macular lesion surrounded by a hyper-autofluorescent halo. (B) Spectral-domain optical coherence tomography of the same eye shows thinning of the retinal pigment epithelium and outer retinal layer, next to the fovea, consistent with geographic atrophy. This figure has been reused with the permission of Med Hypothesis Discov Innov Ophthalmol [16]. 


\section{Dry AMD}

AMD is the most frequent cause of irreversible blindness in developed nations, and its prevalence has been increasing with the aging population [17]. The estimated prevalence of early and late stages of AMD is approximately $8.01 \%$ ( $95 \%$ confidence interval: $3.98-15.49$ ) and $0.37 \%$ (95\% confidence interval: $0.18-0.77$ ), respectively [17]. Late/advanced stages of AMD are characterized by exudation and hemorrhage, as in exudative AMD, or by RPE loss and choriocapillaris, as in dry AMD [18]. Dry AMD accounts for approximately $80 \%-90 \%$ of all advanced AMD cases.

GA is diagnosed when the area of RPE cell loss exceeds $175 \mu \mathrm{m}$ [19]. When it involves the foveal center, profound vision loss occurs. It is usually focal and well-delineated [19]. Atrophy starts as a small area of RPE loss and insidiously increases in dimensions over a few years [19]. This atrophic creep could be related to VEGF signaling, which is both autocrine and paracrine. In the initial stages of dry AMD, downregulation of VEGF production could occur within a solitary RPE cell or group of adjacent RPE cells, resulting in loss of cell survival capability. For compensation, adjacent RPE cells may up-regulate their VEGF production. However, this cannot be sustained, and upon reaching a tipping point, these compensatory RPE cells may become incapable of producing VEGF and undergo atrophy. Over time, this is manifested as enlarging areas of GA.

\section{VEGF}

The polypeptide VEGF has several isoforms, of which VEGF-A, isoform 165, is the most potent. The genetic switch for transcription is located on chromosome 6 [20]. VEGF acts by binding to three transmembrane kinase receptors, VEGFR1, VEGFR2, and VEGFR3 [21]. It promotes angiogenesis under pathological conditions, such as prolonged tissue hypoxia, and maintains cellular function under normal physiologic conditions [22], probably achieved by its background synthesis and secretion by cells, such as RPE cells. It also promotes cell survival by upregulating the expression of the anti-apoptotic proteins BCL2 and A1, inhibitors of apoptotic proteins (IAPs), survivin, and X-linked IAP, which act by suppressing terminal caspases 3 and 7 [23-25]. It enables RPE to scavenge photoreceptor outer segments and maintains the fenestrations of the choriocapillaris and its directional distribution [26].

To date, basal levels of VEGF under normal physiological conditions have not been measured. In the absence of such data, hypo-angiogenesis cannot be investigated through a study of vitreous samples alone. Therefore, to determine basal VEGF quantity and synthesis capacity, other indirect indicators should be measured at the cellular and molecular levels, such as the amount and distribution of VEGF receptors on the RPE and the extent and quality of VEGF synthesis in response to an induction stimulus $[8,26,27]$.

\section{RPE-choriocapillaris complex}

RPE is a single layer of hexagonal cells that supports the retinal photoreceptors across a potential embryonic plane of separation, the subretinal space [28]. It has no regenerative capability and is prone to damage from oxidative stress, as it absorbs most of the incident light impinging on the retina [28]. Each RPE cell supports numerous photoreceptors; therefore, even a small area of RPE loss at the foveal center could lead to severe loss of visual function [28]. Each RPE cell has two distinct regions: the apical and basal regions. While the apical region interacts with photoreceptors through multiple pseudopodal invaginations, the basal region is in contact with the Bruch's membrane and ensures continuous flow of metabolites from the choriocapillaris to the photoreceptors [28]. Thus, the RPE ensures prompt removal of the shed photoreceptor outer segments and provides efficient metabolic exchange [28].

The choriocapillaris is composed of the smallest caliber vessels (Sattler's layer) within the highly vascular choroidal stroma [29]. During embryonic growth, the development of these vessels strongly depends on the $\mathrm{RPE}$, and this process is mediated by VEGF [26]. Maintained by VEGF, choriocapillaris fenestrations are mostly directed toward the RPE [26, 9], ensuring unhindered and rapid nutritional and metabolic supply to the retinal photoreceptors [26].

\section{Interactions among VEGF, RPE, and choriocapillaris}

VEGF secretion from the RPE precedes the development and differentiation of the choriocapillaris in the early embryonic stage [26]. Choriocapillaris development is absent in mice with RPE-specific deletion of VEGF [7, 26]. The RPE actively produces VEGF at low levels, mainly along its basal side, facing the choriocapillaris [3032]. It also has both VEGFR1 and VEGFR2 surface receptors [33]. Destruction of the RPE leads to secondary atrophy of the choriocapillaris, which is thought to be mediated, at least in part, by VEGF derived from the RPE. Autocrine VEGF signaling enhances RPE survival under oxidative stress via the VEGFR2 pathway [8, 33, 
34]. VEGF blockade leads to RPE dysfunction by reduction and blunting of its microvilli and decrease in its ability to phagocytose shed photoreceptor outer segments [35]. Therefore, because of the dual role of directly influencing the formation of the choriocapillaris during developmental stages and promoting survival of the RPE and maintaining the integrity of the choriocapillaris in adults, optimal levels of VEGF are considered vital for ocular homeostasis and integrity [35].

\section{EVALUATION OF THE HYPOTHESIS}

\section{Implications of the hypothesis and potential role for therapeutic angiogenesis}

Oxidative stress is a common risk factor for the pathogenesis of both wet AMD and dry AMD [36]. In wet AMD, VEGF expression is upregulated [36], whereas in dry AMD, it may be downregulated; however, this is yet to be proven. Genetic and epigenetic factors might play a role in determining the molecular milieu of the RPE-Bruch's membrane-choriocapillaris complex, which may impact secondary cellular events, leading to the development of exudative AMD or dry AMD. Owing to the effects of VEGF on RPE cell survival and the choriocapillaris [26], downregulation of VEGF expression could be instrumental in the loss of RPE cells and the choriocapillaris, leading to the development of GA in dry AMD. This remains to be verified in in vitro and in vivo investigations.

Although the exact tissue algorithms remain to be elucidated, it is well-established that VEGF secretion is increased in exudative AMD [36]. Consequently, anti-VEGF therapy for the management of exudative AMD has been successful [37]. However, finding treatment or prophylactic options for GA is crucial because most patients with AMD show the currently untreatable dry AMD that can progress to atrophy of the foveal center, or GA [38]. If the concept of hypo-angiogenesis as a driver for dry AMD is proven, treatment with VEGF or induction of angiogenesis could be considered. Similar attempts at therapeutic angiogenesis have been actively investigated in cardiac and limb ischemia [39-41]. Although pooled data of two clinical trials of the intracoronary administration of the angiogenic therapy product Ad5FGF-4 (alferminogene tadenovec, Generx, Berlex Biosciences, Richmond, California) to induce angiogenesis revealed a gender-specific angiogenic response to the clinical treatment of refractory angina in women [41], the interpretation of results warrant caution for plausible unwanted complications.

\section{CONCLUSIONS}

The reasons for a patient developing exudative AMD or dry AMD remain poorly understood. Nevertheless, targeting increased VEGF production in patients with exudative AMD using anti-VEGF drugs is highly efficacious in preserving vision. Similarly, dry AMD may be a manifestation of reduced VEGF synthesis (hypoangiogenesis) and subsequent decreased RPE cell survival. Experimental studies exploring this possibility could pave the way for clinical trials of angiogenesis to treat dry AMD. In vitro and in vivo assessments of benefits and side effects of this hypothetical treatment are warranted.

\section{ETHICAL DECLARATIONS}

Ethical approval: Not required. Conflict of interests: None

\section{FUNDING}

None.

\section{ACINOWLEGEMENTS}

\section{None.}

\section{REFERENCES}

1. Apte RS, Chen DS, Ferrara N. VEGF in Signaling and Disease: Beyond Discovery and Development. Cell. 2019;176(6):1248-1264. doi: 10.1016/j.cell.2019.01.021 pmid: 30849371

2. CATT Research Group, Martin DF, Maguire MG, Ying GS, Grunwald JE, Fine SL, Jaffe GJ. Ranibizumab and bevacizumab for neovascular age-related macular degeneration. N Engl J Med. 2011;364(20):1897-908. doi: 10.1056/NEJMoa1102673 pmid: 21526923

3. Comparison of Age-related Macular Degeneration Treatments Trials (CATT) Research Group, Maguire MG, Martin DF, Ying GS, Jaffe GJ, Daniel E, Grunwald JE, et al. Five-Year Outcomes with Anti-Vascular Endothelial Growth Factor Treatment of Neovascular 
Age-Related Macular Degeneration: The Comparison of Age-Related Macular Degeneration Treatments Trials. Ophthalmology. 2016;123(8):1751-1761. doi: 10.1016/j.ophtha.2016.03.045 pmid: 27156698

4. Boyer DS, Schmidt-Erfurth U, van Lookeren Campagne M, Henry EC, Brittain C. The Pathophysiology of Geographic Atrophy Secondary to Age-Related Macular Degeneration and The Complement Pathway as a Therapeutic Target. Retina. 2017;37(5):819-835. doi: 10.1097/IAE.0000000000001392 pmid: 27902638

5. Dugel PU, Jaffe GJ, Sallstig P, Warburton J, Weichselberger A, Wieland M, et al. Brolucizumab Versus Aflibercept in Participants with Neovascular Age-Related Macular Degeneration: A Randomized Trial. Ophthalmology. 2017;124(9):1296-1304. doi: 10.1016/j. ophtha.2017.03.057 pmid: 28551167

6. Holz FG, Sadda SR, Busbee B, Chew EY, Mitchell P, Tufail A, et al; Chroma and Spectri Study Investigators. Efficacy and Safety of Lampalizumab for Geographic Atrophy Due to Age-Related Macular Degeneration: Chroma and Spectri Phase 3 Randomized Clinical Trials. JAMA Ophthalmol. 2018;136(6):666-677. doi: 10.1001/jamaophthalmol.2018.1544 pmid: 29801123

7. Ford KM, Saint-Geniez M, Walshe T, Zahr A, D'Amore PA. Expression and role of VEGF in the adult retinal pigment epithelium. Invest Ophthalmol Vis Sci. 2011 9;52(13):9478-87. doi: 10.1167/iovs.11-8353 pmid: 22058334

8. Saint-Geniez M, Kurihara T, Sekiyama E, Maldonado AE, D'Amore PA. An essential role for RPE-derived soluble VEGF in the maintenance of the choriocapillaris. Proc Natl Acad Sci U S A. 2009;106(44):18751-6. doi: 10.1073/pnas.0905010106 pmid: 19841260

9. Saint-Geniez M, Maldonado AE, D'Amore PA. VEGF expression and receptor activation in the choroid during development and in the adult. Invest Ophthalmol Vis Sci. 2006;47(7):3135-42. doi: 10.1167/iovs.05-1229 pmid: 16799060

10. Lee S, Chen TT, Barber CL, Jordan MC, Murdock J, Desai S, et al. Autocrine VEGF signaling is required for vascular homeostasis. Cell. 2007;130(4):691-703. doi: 10.1016/j.cell.2007.06.054 pmid: 17719546

11. Clare AJ, Liu J, Copland DA, Theodoropoulou S, Dick AD. Unravelling the therapeutic potential of IL-33 for atrophic AMD. Eye (Lond). 2021. doi: 10.1038/s41433-021-01725-5 pmid: 34531552

12. Ambati J, Atkinson JP, Gelfand BD. Immunology of age-related macular degeneration. Nat Rev Immunol. 2013;13(6):438-51. doi: 10.1038/nri3459 pmid: 23702979

13. Grunwald JE, Daniel E, Huang J, Ying GS, Maguire MG, Toth CA, et al; CATT Research Group. Risk of geographic atrophy in the comparison of age-related macular degeneration treatments trials. Ophthalmology. 2014;121(1):150-161. doi: 10.1016/j. ophtha.2013.08.015 pmid: 24084496

14. Grunwald JE, Pistilli M, Ying GS, Maguire MG, Daniel E, Martin DF; Comparison of Age-related Macular Degeneration Treatments Trials Research Group. Growth of geographic atrophy in the comparison of age-related macular degeneration treatments trials. Ophthalmology. 2015;122(4):809-16. doi: 10.1016/j.ophtha.2014.11.007 pmid: 25542520

15. Gemenetzi M, Lotery AJ, Patel PJ. Risk of geographic atrophy in age-related macular degeneration patients treated with intravitreal antiVEGF agents. Eye (Lond). 2017;31(1):1-9. doi: 10.1038/eye.2016.208 pmid: 27716750

16. Massamba N, Sellam A, Butel N, Skondra D, Caillaux V, Bodaghi B. Use of Fundus Autofluorescence Combined with Optical Coherence Tomography for Diagnose of Geographic Atrophy in Age-Related Macular Degeneration. Med Hypothesis Discov Innov Ophthalmol. 2019;8(4):298-305. pmid: 31788492

17. Wong WL, Su X, Li X, Cheung CM, Klein R, Cheng CY, et al. Global prevalence of age-related macular degeneration and disease burden projection for 2020 and 2040: a systematic review and meta-analysis. Lancet Glob Health. 2014;2(2):e106-16. doi: 10.1016/S2214109X(13)70145-1 pmid: 25104651

18. Keenan TDL, Cukras CA, Chew EY. Age-Related Macular Degeneration: Epidemiology and Clinical Aspects. Adv Exp Med Biol. 2021;1256:1-31. doi: 10.1007/978-3-030-66014-7_1 pmid: 33847996

19. Fleckenstein M, Mitchell P, Freund KB, Sadda S, Holz FG, Brittain C, et al. The Progression of Geographic Atrophy Secondary to AgeRelated Macular Degeneration. Ophthalmology. 2018;125(3):369-390. doi: 10.1016/j.ophtha.2017.08.038 pmid: 29110945

20. Holmes DI, Zachary I. The vascular endothelial growth factor (VEGF) family: angiogenic factors in health and disease. Genome Biol. 2005;6(2):209. doi: 10.1186/gb-2005-6-2-209 pmid: 15693956

21. Karamysheva AF. Mechanisms of angiogenesis. Biochemistry (Mosc). 2008;73(7):751-62. doi: 10.1134/s0006297908070031 pmid: 18707583

22. Takahashi H, Shibuya M. The vascular endothelial growth factor (VEGF)/VEGF receptor system and its role under physiological and pathological conditions. Clin Sci (Lond). 2005;109(3):227-41. doi: 10.1042/CS20040370 pmid: 16104843

23. Saleem M, Qadir MI, Perveen N, Ahmad B, Saleem U, Irshad T, et al. Inhibitors of apoptotic proteins: new targets for anticancer therapy Chem Biol Drug Des. 2013;82(3):243-51. doi: 10.1111/cbdd.12176 pmid: 23790005

24. Mesri M, Morales-Ruiz M, Ackermann EJ, Bennett CF, Pober JS, Sessa WC, et al. Suppression of vascular endothelial growth factormediated endothelial cell protection by survivin targeting. Am J Pathol. 2001;158(5):1757-65. doi: 10.1016/S0002-9440(10)64131-4 pmid: 11337373

25. Maier JK, Lahoua Z, Gendron NH, Fetni R, Johnston A, Davoodi J, et al. The neuronal apoptosis inhibitory protein is a direct inhibitor of caspases 3 and 7. J Neurosci. 2002;22(6):2035-43. doi: 10.1523/JNEUROSCI.22-06-02035.2002 pmid: 11896143

26. Marneros AG, Fan J, Yokoyama Y, Gerber HP, Ferrara N, Crouch RK, et al. Vascular endothelial growth factor expression in the retinal pigment epithelium is essential for choriocapillaris development and visual function. Am J Pathol. 2005;167(5):1451-9. doi: 10.1016/ S0002-9440(10)61231-X pmid: 16251428

27. Mateos L, Perez-Alvarez MJ, Wandosell F. Angiotensin II type-2 receptor stimulation induces neuronal VEGF synthesis after cerebral ischemia. Biochim Biophys Acta. 2016;1862(7):1297-308. doi: 10.1016/j.bbadis.2016.03.013 pmid: 27045356

28. Boulton M, Dayhaw-Barker P. The role of the retinal pigment epithelium: topographical variation and ageing changes. Eye (Lond). 2001;15(Pt 3):384-9. doi: 10.1038/eye.2001.141 pmid: 11450762

29. Nickla DL, Wallman J. The multifunctional choroid. Prog Retin Eye Res. 2010;29(2):144-68. doi: 10.1016/j.preteyeres.2009.12.002 pmid: 20044062

30. Bhutto IA, McLeod DS, Hasegawa T, Kim SY, Merges C, Tong P, et al. Pigment epithelium-derived factor (PEDF) and vascular endothelial growth factor (VEGF) in aged human choroid and eyes with age-related macular degeneration. Exp Eye Res. 2006;82(1):99- 
110. doi: 10.1016/j.exer.2005.05.007 pmid: 16019000

31. Witmer AN, Vrensen GF, Van Noorden CJ, Schlingemann RO. Vascular endothelial growth factors and angiogenesis in eye disease. Prog Retin Eye Res. 2003;22(1):1-29. doi: 10.1016/s1350-9462(02)00043-5 pmid: 12597922

32. George SM, Lu F, Rao M, Leach LL, Gross JM. The retinal pigment epithelium: Development, injury responses, and regenerative potential in mammalian and non-mammalian systems. Prog Retin Eye Res. 2021;85:100969. doi: 10.1016/j.preteyeres.2021.100969 pmid: 33901682

33. Byeon SH, Lee SC, Choi SH, Lee HK, Lee JH, Chu YK, et al. Vascular endothelial growth factor as an autocrine survival factor for retinal pigment epithelial cells under oxidative stress via the VEGF-R2/PI3K/Akt. Invest Ophthalmol Vis Sci. 2010;51(2):1190-7. doi: 10.1167/iovs.09-4144 pmid: 19834034

34. Klettner A, Möhle F, Roider J. Intracellular bevacizumab reduces phagocytotic uptake in RPE cells. Graefes Arch Clin Exp Ophthalmol. 2010;248(6):819-24. doi: 10.1007/s00417-010-1317-x pmid: 20169356

35. Bonilha VL, Rayborn ME, Saotome I, McClatchey AI, Hollyfield JG. Microvilli defects in retinas of ezrin knockout mice. Exp Eye Res. 2006;82(4):720-9. doi: 10.1016/j.exer.2005.09.013 pmid: 16289046

36. Zarbin MA. Current concepts in the pathogenesis of age-related macular degeneration. Arch Ophthalmol. 2004;122(4):598-614. doi: 10.1001/archopht.122.4.598 pmid: 15078679

37. Parravano M, Costanzo E, Scondotto G, Trifirò G, Virgili G. Anti-VEGF and Other Novel Therapies for Neovascular Age-Related Macular Degeneration: An Update. BioDrugs. 2021;35(6):673-692. doi: 10.1007/s40259-021-00499-2 pmid: 34655434

38. GBD 2019 Blindness and Vision Impairment Collaborators; Vision Loss Expert Group of the Global Burden of Disease Study. Causes of blindness and vision impairment in 2020 and trends over 30 years, and prevalence of avoidable blindness in relation to VISION 2020: the Right to Sight: an analysis for the Global Burden of Disease Study. Lancet Glob Health. 2021;9(2):e144-e160. doi: 10.1016/S2214109X(20)30489-7 Erratum in: Lancet Glob Health. 2021;9(4):e408. pmid: 33275949

39. Giacca M, Zacchigna S. VEGF gene therapy: therapeutic angiogenesis in the clinic and beyond. Gene Ther. 2012;19(6):622-9. doi: 10.1038/gt.2012.17 pmid: 22378343

40. Losordo DW, Dimmeler S. Therapeutic angiogenesis and vasculogenesis for ischemic disease: part II: cell-based therapies. Circulation. 2004;109(22):2692-7. doi: 10.1161/01.CIR.0000128596.49339.05 pmid: 15184293

41. Henry TD, Grines CL, Watkins MW, Dib N, Barbeau G, Moreadith R, et al. Effects of Ad5FGF-4 in patients with angina: an analysis of pooled data from the AGENT-3 and AGENT-4 trials. J Am Coll Cardiol. 2007;50(11):1038-46. doi: 10.1016/j.jacc.2007.06.010 pmid: 17825712 